Nuclear Physics A423 (1984) 77-92

(C) North-Holland Publishing Company

\title{
FIXED-JT AVERAGES OF ELECTROMAGNETIC OPERATORS
}

\author{
J.J.M. VERBAARSCHOT* and P.J. BRUSSAARD \\ Fysisch Laboratorium, Postbus 80.000, 3508 TA Utrecht, The Netherlands
}

Received 23 June 1983

(Revised 5 December 1983)

\begin{abstract}
A method is presented to express fixed- $J T$ traces in terms of fixed $J_{z} T_{z}$ traces for an arbitrary tensor operator. The method is used to evaluate the cueflicients of the linear, secular energy variation of the electric quadrupole moment and the gyromagnetic ratio for a number of nuclei in the sd shell. Upper limits for the electromagnetic moments are used to estimate the fluctuations about this secular variation.
\end{abstract}

\section{Introduction}

In order to obtain the electromagnetic moments of nuclear states in a shell-model calculation, one has to evaluate the electromagnetic transition operator taken between two identical eigenstates. The required eigenfunctions can be derived from a diagonalization of the hamiltonian in an appropriate configuration space. In most cases this space is determined by the size of the matrices that can be diagonalized by a computer. In order to perform calculations in larger spaces one has to look for different methods. One of these methods is statistical spectroscopy ${ }^{1-3}$ ). The idea behind this method is that the relevant nuclear properties are obtained by the calculation of averages of operators in a given configuration space. The averages are given, essentially, by the traces of those operators which can be evaluated directly.

In the case of electromagnetic transition amplitudes it was shown by Draayer $e t$ $a l^{7,15}$ ) that the amplitudes could be described locally in the space of the initial and final energies by a gaussian distribution (Porter-Thomas assumption). The variance of this distribution showed a secular behaviour (as a function of the initial and final energies) that could be well described by low-order polynomials with coefficicnts that could be expressed in terms of moments of the hamiltonian and the electromagnetic operator.

In this paper we study the case of electromagnetic moments where the situation is more complicated. In general the electromagnetic moments do not fluctuate about zero, but show a nontrivial secular variation which depends on the angular momentum and isospin of the states involved. It is no use to describe the fluctuations by means of the Porter-Thomas assumption because the variance of the diagonal

* Present address: Max-Planck-Institut für Kernphysik, Postfach 103980, D-6900 Heidelberg 1, Federal Republic of Germany. 
elements of an operator cannot be expressed in terms of the moments of the hamiltonian and the electromagnetic operator. Hence they cannot be calculated without the explicit use of shell-model wave functions.

In sect. 2 we present some shell-model results which clearly show the nature of the fluctuations. In sect. 3 we evaluate the properties of the electromagnetic moments without using explicit shell-model wave functions.

As is discussed in sect. 3 the secular behaviour can be derived from fixed-JT traces of a tensor operator. Let us disregard isospin for the moment. The fixed- $J$ traces can be expressed in traces for a fixed value of the angular-momentum projection $J_{z}$. The latter traces can be evaluated by taking particle partitions over sets of single-particle states all of which have the same angular-momentum projection $^{4,5}$ ). The required propagation polynomials can be expressed in terms of the number of states for fixed angular-momentum projection ${ }^{6}$ ). Although for a scalar operator it is almost trivial to express traces for fixed $J$ in terms of the traces for fixed $J_{z}$, in the case of an arbitrary tensor operator the direct application of the usual relations leads to a lengthy calculation. A concise method to derive traces for fixed $J$ from the fixed- $J_{z}$ traces of an arbitrary tensor operator is developed in appendix A. Sect. 3 concludes with a discussion of the numerical results for a few nuclei in the sd shell.

In sect. 4 the magnitude of the fluctuations is estimated by the calculation of upper limits. Both scalar and $J$-dependent upper limits are studied and compared with the shell-model results. Better estimates for the largest moments are obtained with statistical assumptions for random-matrix ensembles. Concluding remarks are made in sect. 5 .

\section{Shell-model results}

The electric quadrupole moments and magnetic dipole moments ${ }^{9}$ ) of the nuclides ${ }^{20} \mathrm{Ne},{ }^{21} \mathrm{Ne},{ }^{24} \mathrm{Mg}$ and ${ }^{28} \mathrm{Si}$ have been calculated with the shell model for the complete sd shell. The surface delta interaction was used. The single-particle energies were taken to be $\varepsilon\left(0 \mathrm{~d}_{5 / 2}\right)=-4.15 \mathrm{MeV}, \varepsilon\left(1 \mathrm{~s}_{1 / 2}\right)=-3.28 \mathrm{MeV}$ and $\varepsilon\left(0 \mathrm{~d}_{3 / 2}\right)=0.88 \mathrm{MeV}$.

Some typical examples of the calculated results for the electric quadrupole moments and the magnetic dipole moments are shown in figs. 1a, 1b, 2a and 2b. The secular behaviour will be assumed to be linear. Owing to the large fluctuations one can do no better. In general one can say that the quadrupole moments fluctuate about zero for low-spin values. The magnetic moments show, when plotted versus the energy, a slope that is almost independent of $J$ for each nuclide. The average of the calculated values turns out to be very close to the Schmidt value.

The fluctuations about the secular behaviour are rather large, i.e. of the same order of magnitude as the secular variation over the whole spectrum. In particular in the ground-state region one can find large fluctuations for the isoscalar quadrupole moment. 

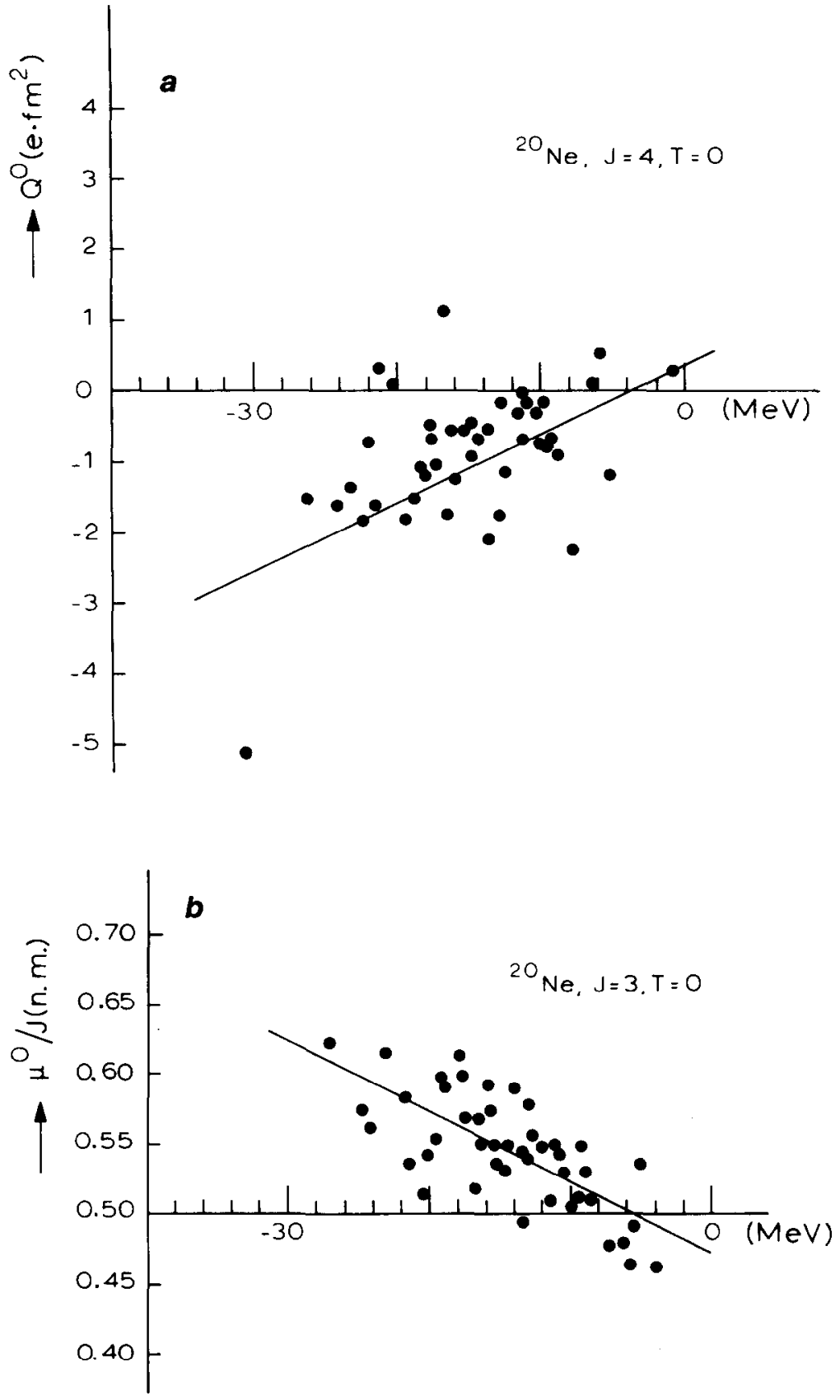

Fig. 1. Exact shell-model results for the isoscalar quadrupole moment $O^{\circ}(\mathrm{a})$ and the isoscalar magnetic moment $\mu^{\circ}$ (b) as a function of the excitation energy of ${ }^{20} \mathrm{Ne}, T=0$ with angular-momentum values as indicated. The straight line represents the linear secular behaviour with coefficients given in figs. 3 and 4. 

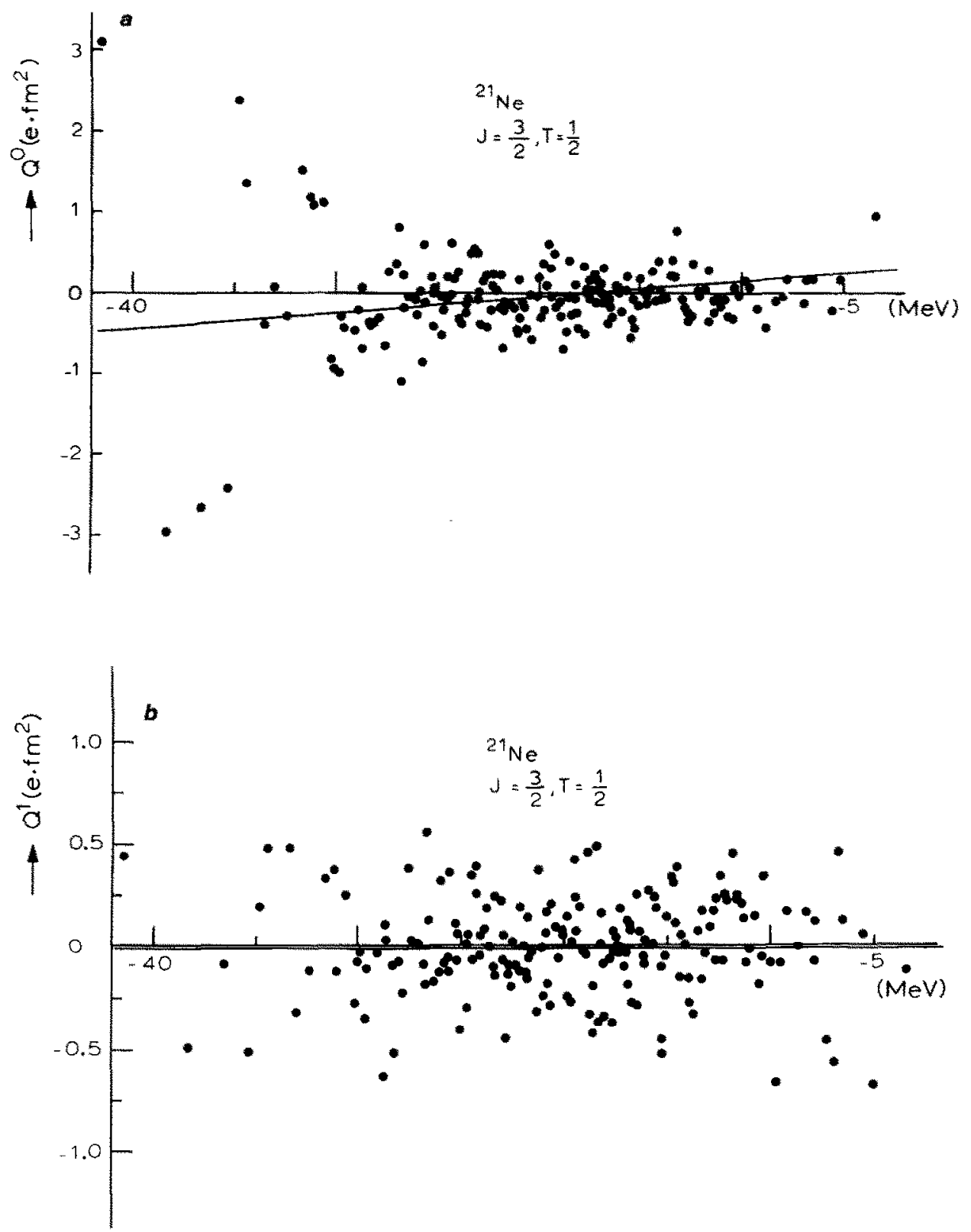

Fig. 2. Exact shell-model results for the isoscalar quadrupole moment $Q^{\circ}(a)$ and isovector quadrupole moment $Q^{1}$ (b) as a function of the excitation energy of ${ }^{21} \mathrm{Ne}, J=\frac{3}{2}, T=\frac{1}{2}$. The straight line represents the linear secular behaviour with coefficients given in figs. 3 and 4 . 
Concluding one can say that only for large $J$-values can a clear secular behaviour be observed. In other cases the electromagnetic moments show a purely stochastic behaviour (possibly with the exception of one or two low-lying states) with the magnitude of the fluctuations as the most relevant quantity. The wave functions do not seem to contain any specific information and the use of an explicit calculation might be questioned.

In the following sections it will be shown that the secular behaviour and the magnitude of the fluctuations can also be evaluated without explicit calculation of the shell-model wave function.

\section{Evaluation of the coefficients of the secular behaviour}

The energy dependence of the expectation values of an electromagnetic operator $O^{\lambda}$ can be obtained from an expansion $\left.{ }^{7}\right)$ of the $\delta$-function $\delta(H-E)$ in polynomials $P_{\nu}(H)$ and $P_{\nu}(E)$ which constitute an orthonormal system of functions associated with the eigenvalue density as a weight function, i.e.

$$
O^{\lambda}(E, n, J)=\left\langle O^{\lambda} \delta(H-E)\right\rangle_{n J}=\sum_{\nu}\left\langle O^{\lambda} P_{\nu}(H)\right\rangle_{n J} P_{\nu}(E)
$$

with the expectation value

$$
\langle A\rangle_{n J}=\frac{1}{d_{n J}} \operatorname{Tr}_{n J}(A)
$$

Here $d_{n J}$ denotes the dimension of the $n$-particle space of spin $J$. It is assumed that the central-limit theorem operates such that the eigenvalues $E_{i}$ of $H$ and also $E_{i}+\varepsilon O^{\lambda}\left(E_{i}\right)(\varepsilon$ small $)$ possess a gaussian distribution, i.e. the higher-order cumulants are zero for the set $\left\{E_{i}\right\}$ and zero up to first order in $\varepsilon$ for the set $\left\{E_{i}+\varepsilon O^{\lambda}\left(E_{i}\right)\right\}\left[\right.$ refs. $\left.\left.{ }^{7,8}\right)\right]$. The two distributions must be identical to within a scale change and hence $O^{\lambda}(E)$ must show a linear dependence on $E$. If in eq. (3.1) only the terms $\nu=0$ and $\nu=1$ are retained, the energy dependence is given by

$$
O^{\lambda}(E, n, J)=\left\langle O^{\lambda}\right\rangle_{n J}+\frac{\left\langle O^{\lambda}\left(H-E_{n J}\right)\right\rangle_{n J}\left(E-E_{n J}\right)}{\sigma_{n J}^{2}},
$$

where $F_{n J}$ and $\sigma_{n J}^{2}$ are the centroid and variance of the considered eigenvalue distribution of $H$, respectively.

In appendix $\mathrm{A}$ it is shown that for fixed values of $J$ the expectation value of the tensor operator in eq. (3.3) can be expressed, as was discussed in the introduction, in terms of traces for fixed values of $J_{z}$. The latter can be evaluated by the use of a contraction procedure that leads to a diagrammatic expansion ${ }^{6}$ ) which describes the way the operators have been contracted. In the present paper the neutron-proton formalism is used rather than the isospin formalism. Thus one obtains for the traces 
relating to eq. (3.3) the expansions

$$
\operatorname{Tr}_{n_{\mathrm{n}} n_{\mathrm{p}} J_{z}}\left(O_{0}^{\lambda} H_{2}\right)=\sum_{\omega_{1} \omega_{2} \omega_{3}} \operatorname{Tr}_{n_{\mathrm{n}} n_{\mathrm{p}} J_{z}}\left(O_{0}^{\lambda}\right)=\sum_{\omega}
$$

where $H_{1}$ and $H_{2}$ are the one- and two-body parts of the hamiltonian, respectively. The index $\omega_{i}$ tells whether the contraction applies to a proton $(\pi)$ or a neutron $(\nu)$ orbit. As an example an explicit expression for the following diagram is given:
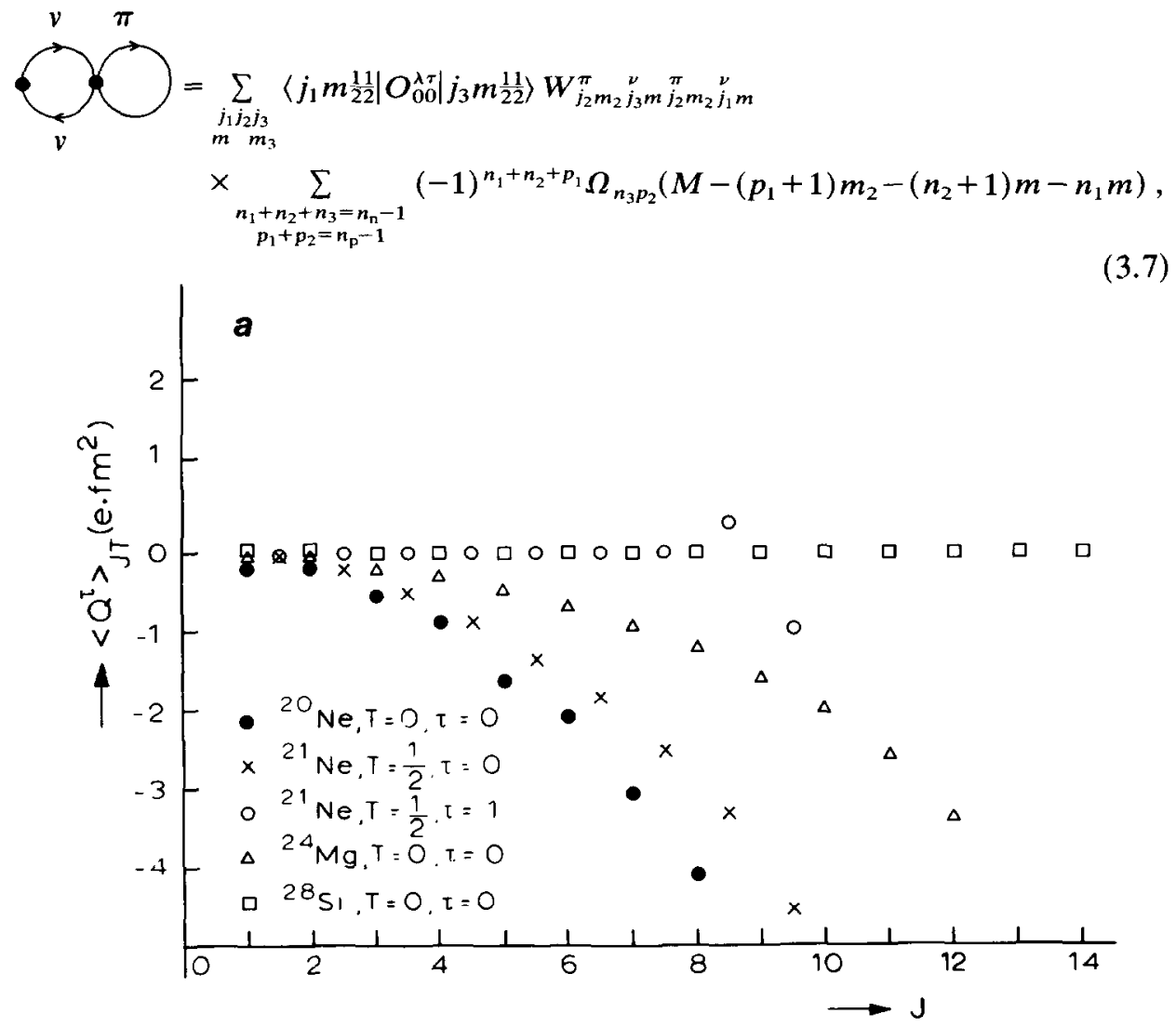

Fig. 3. The average values of the isoscalar $(\tau=0)$ and isovector $(\tau=1)$ quadrupole moment $\left\langle Q^{\tau}\right\rangle_{J T}$ (a) and the slope $\left\langle Q^{\tau}\left(H-E_{J T}\right)\right\rangle_{J T} / \sigma_{J T}(\mathrm{~b})$ as a function of the angular momentum $J$ for ${ }^{20} \mathrm{Ne},{ }^{24} \mathrm{Mg},{ }^{28} \mathrm{Si}$ $(T=0)$ and for ${ }^{21} \mathrm{Ne}\left(T=\frac{1}{2}\right)$. 


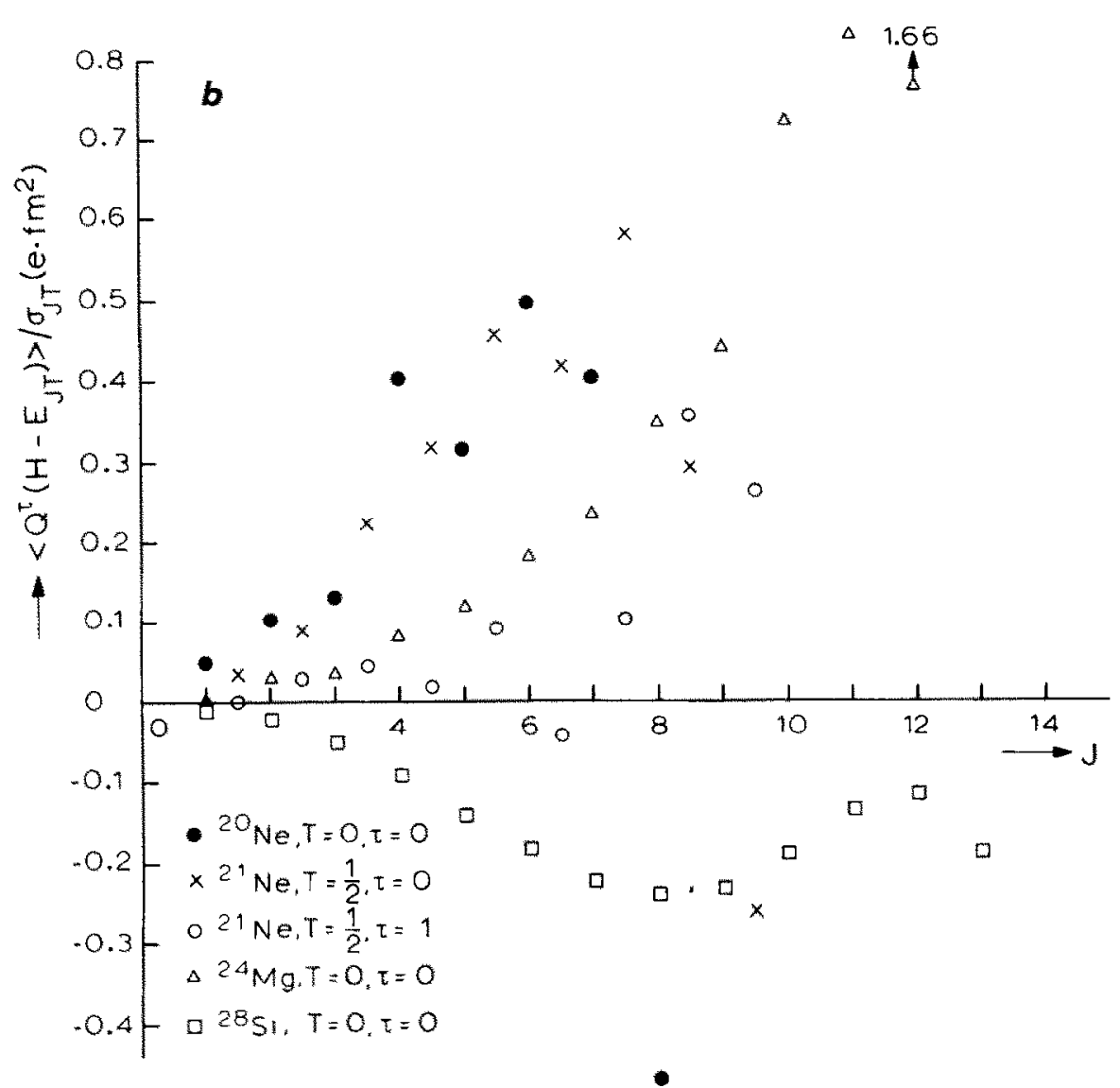

Fig. 3.-(contd.)

where $W_{\alpha \beta \gamma \delta}$ represents the antisymmetrized two-body matrix elements and $\Omega_{n p}(M)$ the number of states for $n$ neutrons and $p$ protons for given angular-momentum projection $M$.

The angular-momentum dependence of the average quadrupole moment $\left\langle Q^{\tau}\right\rangle_{J T}$ and of the correlation between the quadrupole moment and the hamiltonian $\left\langle Q^{\tau}\left(H-E_{J T}\right)\right\rangle_{J T} / \sigma_{J T}$ are given in figs. $3 \mathrm{a}$ and $3 \mathrm{~b}$. The centroid and the width of the eigenvalue distribution of the hamiltonian for fixed angular momentum $J$ and isospin $T$ are denoted by $E_{J T}$ and $\sigma_{J T}$. In the case of ${ }^{28} \mathrm{Si}$ the average quadrupole moment is zero, which is a consequence of the minus sign under particle-hole transformation for even-order single-particle tensor operators ${ }^{10,11}$ ). For the upper part of the sd shell, one finds an increasing value of the quadrupole moment as a function of the angular momentum. The angular-momentum dependence of the correlation $\left(Q^{\top}\left(H-E_{y T}\right)\right\rangle_{J T} / \sigma_{J T}$ shows a relatively irregular pattern. In general one finds larger values of the correlation for larger angular momenta. 
The matrix element of the $z$-component of the isoscalar M1 operator can be written as

$$
\mu^{0} \equiv\left\langle J J_{z}=J T\left|\mu_{z}^{0}\right| J J_{z}=J T\right\rangle=\frac{1}{2} J+\frac{1}{2}\left(g^{s}-1\right)\left\langle J J_{z}=J T\left|S_{z}\right| J J_{z}=J T\right\rangle,
$$

with $g^{s}$ the isoscalar spin $g$-factor and $S_{z}$ the $z$-component of the total intrinsic spin. We shall consider the energy dependence of $\mu^{\%} / J$. The $J$-dependence of the average isoscalar magnetic momentum $\left\langle\mu_{z}^{0}\right\rangle_{J \mathrm{~F}}$ divided by $J$ and of the correlation $\left\langle\mu_{z}^{0}\left(H-E_{J T}\right)\right\rangle_{J T} / \sigma_{J T}$ divided by $J$ are given in figs. $4 \mathrm{a}$ and $4 \mathrm{~b}$, respectively. The quotient of the average isoscalar moment and $J$ appears to be almost independent of the angular momentum $J$ and is slightly smaller than the $j=\frac{5}{2}$ single-shell value of $0.576 \mathrm{n} . \mathrm{m}$. The correlation of the average isoscalar magnetic moment and the
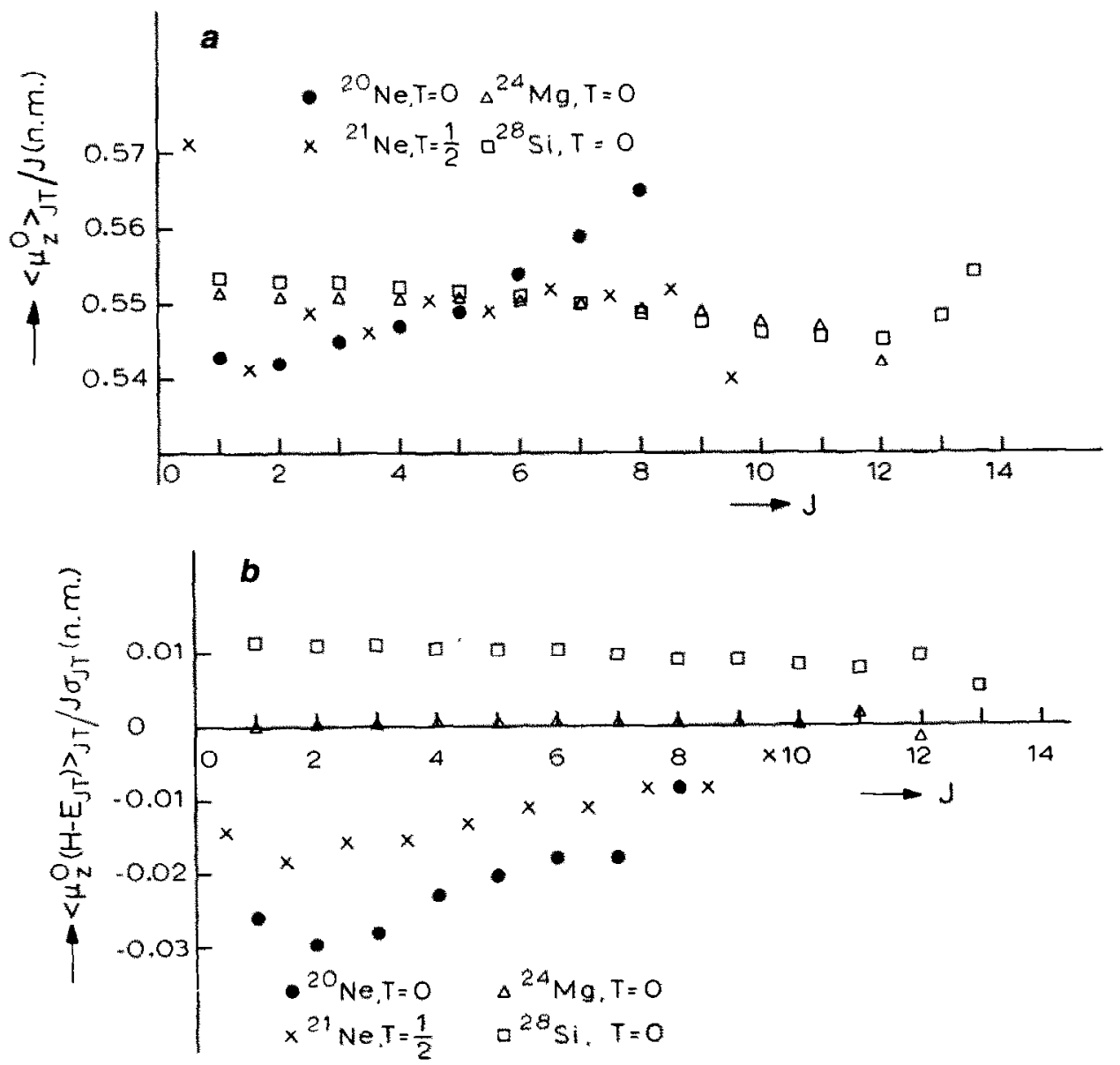

Fig. 4. The average values of the isoscalar magnetic moment ratio $\left\langle\mu_{2}^{0}\right\rangle_{J T} / J$ (a) and the slope $\left\langle\mu_{z}^{0}\left(H-E_{J T}\right)\right\rangle_{J T} / J \sigma_{J T}(\mathrm{~b})$ as a function of the angular momentum $J$ for ${ }^{20} \mathrm{Ne},{ }^{24} \mathrm{Mg},{ }^{28} \mathrm{Si}(T=0)$ and ${ }^{2 i} \mathrm{Ne}\left(T=\frac{1}{2}\right)$. 
hamiltonian, divided by $J$, does not depend strongly on the angular momentum. In the case of ${ }^{24} \mathrm{Mg}$ the correlation is remarkably close to zero. This suggests that one can estimate the $J$-dependence by means of scalar moments of the hamiltonian and the magnetic dipole operator. The correlations of the electric quadrupole operator and the hamiltonian, which show an irregular behaviour as a function of $J$, can only be obtained [in contrast to e.g. ref. ${ }^{16}$ ) where scalar moments are used] by exact, fixed- $J T$ averaging.

It is seen that the conclusions that were made in sect. 2 from a few shell-model calculations are now confirmed for many more cases by the use of a moment method. These more general results are thus obtained in an efficient way, but the fluctuations about the averages cannot be reproduced.

In the next section the magnitude of the fluctuations will be considered and upper limits will be presented.

\section{Upper limits for the magnitude of the fluctuations}

A crude estimate of an upper limit of electromagnetic moments $\left(\omega_{\text {max }}\right)$ in a space with a definite value of angular momentum and isospin can be obtained from the square root of the total strength and the appropriate geometrical factor,

$$
\omega_{\max }=\left[\sum_{\alpha \alpha^{\prime}}\left\langle J T T_{z} \alpha\left\|O_{0}^{\lambda \tau}\right\| J T T_{z} \alpha^{\prime}\right\rangle^{2}\left(\begin{array}{ccc}
J & \lambda & J \\
-J & 0 & J
\end{array}\right)^{2}\right]^{1 / 2} .
$$

This upper limit is interaction independent and depends on the size of the model space. For the isoscalar magnetic moment an upper limit is calculated for the spin operator (cf. eq. (3.8)).

The total strength can be written as a fixed- $J T$ trace ${ }^{12}$ ) and subsequently evaluated as indicated above.

In tables 1 and 2 results are shown for quadrupole moments and isoscalar magnetic moments of ${ }^{20} \mathrm{Ne},{ }^{21} \mathrm{Ne},{ }^{24} \mathrm{Mg}$ and ${ }^{28} \mathrm{Si}$ in the sd shell. Comparison with the experimental value shows that the calculated upper limit for the quadrupole moment of the $J=2, T=0$ states of ${ }^{20} \mathrm{Ne}$ is rather close.

An alternative way to obtain an upper limit is to calculate for the hermitean one-body operator $O_{00}^{\lambda \tau}$ the eigenvalue $\Omega_{\max }$ with maximum absolute value. This can be performed by taking the $n$th root of the trace of the $n$th power ( $n$ even) of $U_{00}^{\lambda}$ in a space with $n_{\mathrm{n}}$ neutrons and $n_{\mathrm{p}}$ protons:

$$
\left|\Omega_{\max }\right|=\lim _{\substack{n \rightarrow \infty \\ n \text { even }}}\left[\operatorname{Tr}_{n_{n} n_{\mathrm{p}}}\left(O_{00}^{\lambda \tau}\right)^{n}\right]^{1 / n} \leqslant\left[\operatorname{Tr}_{n_{n} n_{\mathrm{p}}}\left(O_{\mathrm{OO}}^{\lambda \tau}\right)^{n}\right]^{1 / n} \equiv \omega_{\max }^{(n)},
$$

where the inequality applies for any finite (even) value of $n$. It will be used to estimate $\Omega_{\max }$. In the case of an isoscalar operator the traces are evaluated in a space of fixed isospin and in the case of an isovector operator the traces are evaluated 


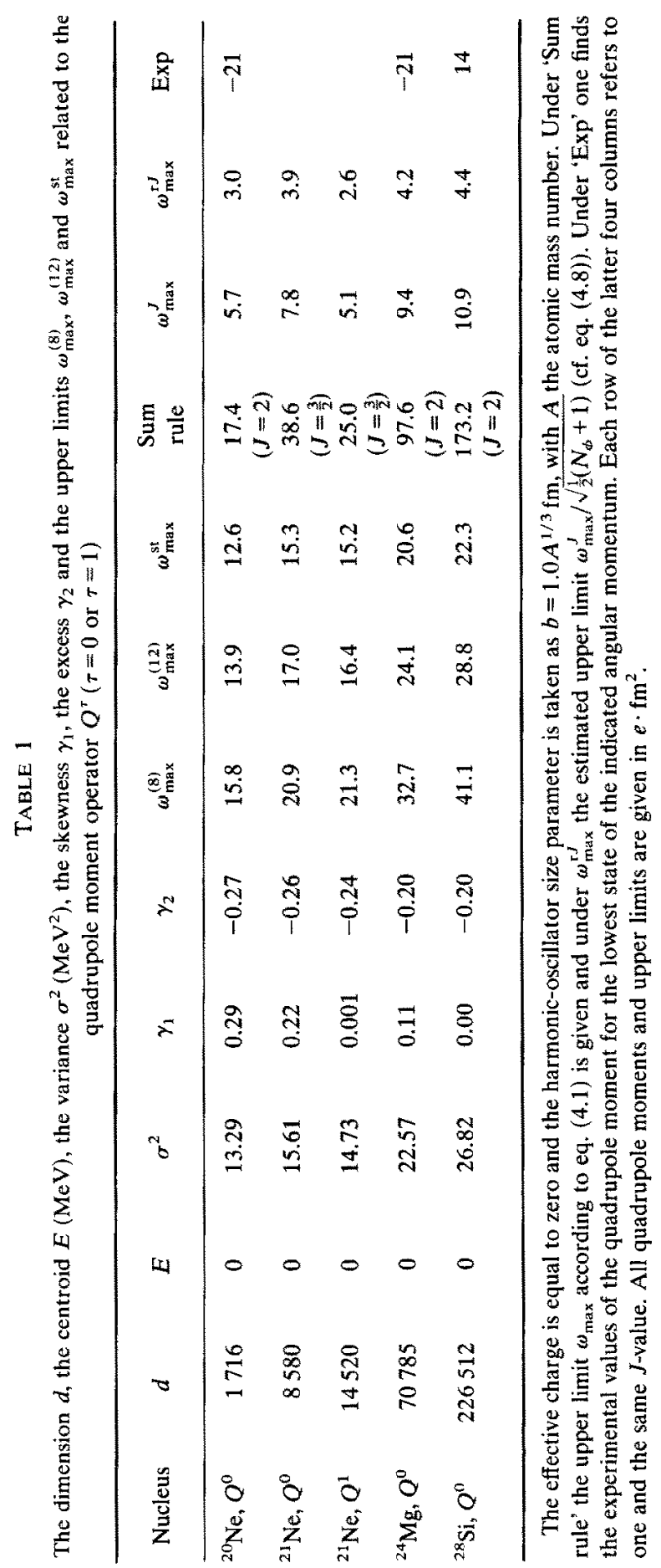




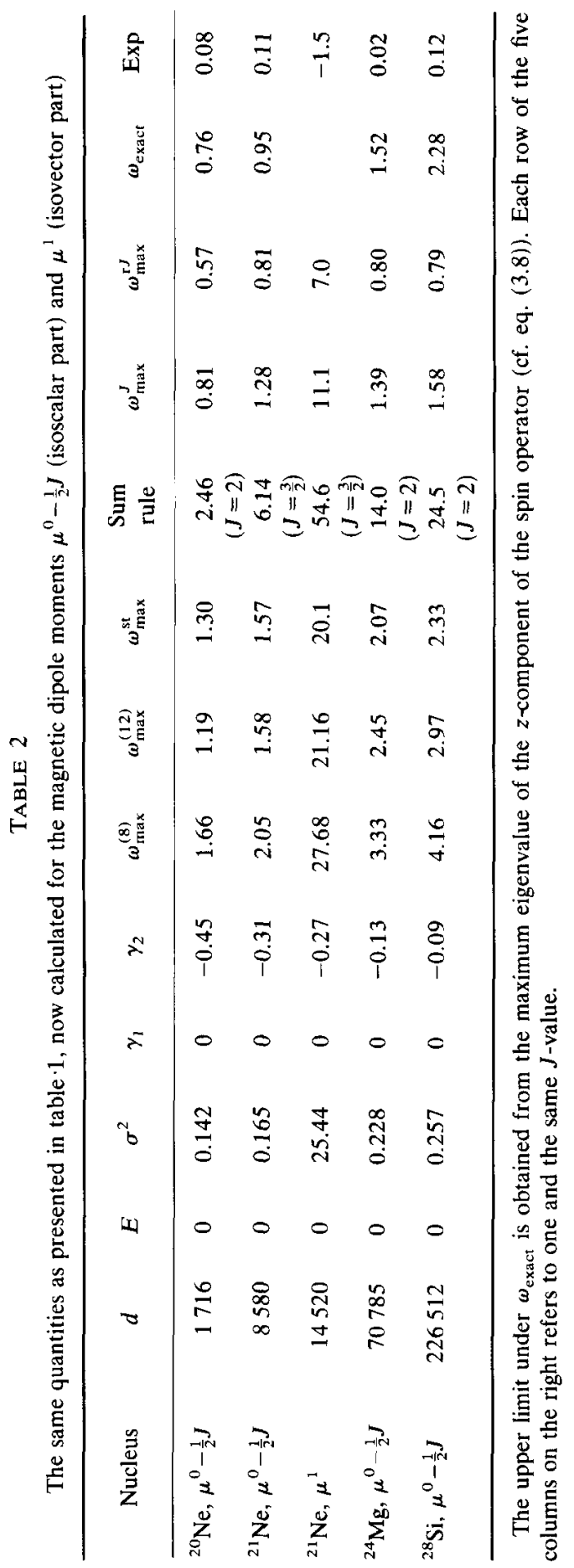


in a space of fixed isospin projection. In this way upper limits are obtained which must hold for all $J$-values. As the value of the electromagnetic moments tends to increase for large $J$-values (see sect. 3 ) one will thus obtain too large upper limits for the lower $J$-values.

The parameters of the distribution function $\rho(\omega)$ of the eigenvalues of $O_{00}^{\lambda \tau}$ are also determined by $\operatorname{Tr}_{n_{\mathrm{n}} n_{\mathrm{p}}}\left(\left(O_{00}^{\lambda \tau}\right)^{p}\right)$. The third and fourth moments show the deviation from a gaussian distribution. An estimate of the extreme eigenvalue $\omega_{\max }$ can be derived from the condition

$$
\int_{\omega_{\max }^{\mathrm{st}}}^{\infty} \rho(\omega) \mathrm{d} \omega=\frac{1}{2}
$$

When the centroid of $\rho(\omega)$ is positive or zero (which is true in all cases that have been considered) this value of $\omega_{\max }^{\text {st }}$ can be used to check the convergence in eq. (4.2) for any finite (even) value of $n$.

The moments for $n_{\mathrm{n}}$ neutrons and $n_{\mathrm{p}}$ protons in the sd shell can be calculated by an extension of Ginocchio's single-orbit result [ ref. $^{13}$ ), eq. (55b)].

Calculations have been performed for the magnetic dipole moments and the electric quadrupole moments of ${ }^{20} \mathrm{Ne},{ }^{21} \mathrm{Ne},{ }^{24} \mathrm{Mg}$ and ${ }^{28} \mathrm{~S}$ in the sd shell. The results of the first five, the 8th and the 12th moments are shown in tables 1 and 2. The centroid $E$, the width $\sigma$, the skewness $\gamma_{1}$ and the excess $\gamma_{2}$ have been derived from the first four moments. The upper limits $\omega_{\max }^{(8)}$ and $\omega_{\max }^{(12)}$ follow from the 8 th and 12 th moments (cf. eq. (4.2)). The small values of $\gamma_{1}$ and $\gamma_{2}$ show that the distribution function $\rho(\omega)$ of the eigenvalues of $O_{00}^{\lambda \tau}$ is very close to gaussian. All odd moments of the magnetic moment operator are zero because the operator is the sum of the operators $l_{z}$ and $s_{z}$. By using particle-hole symmetry it can be shown that the odd moments of the quadrupole moment operator for ${ }^{28} \mathrm{Si}$ are zero. The centroid $\operatorname{Tr}_{n_{\mathrm{n}} n_{\mathrm{p}}}\left(O_{00}^{\lambda \tau}\right)$ is always equal to zero for $\lambda \neq 0$. This is a consequence of the vanishing single-particle average,

$$
\sum_{m}\left\langle j m\left|O_{\mathrm{Oo}}^{\lambda \tau}\right| j m\right\rangle=\sum_{m}(-1)^{j-m}\left(\begin{array}{ccc}
j & \lambda & j \\
-m & 0 & m
\end{array}\right)\left\langle j\left\|O_{0}^{\lambda \tau}\right\| j\right\rangle=0 \text { for } \lambda \neq 0 .
$$

Comparison of the values for the upper limits obtained from eq. (4.2) shows that for $n=12$ the convergence is rather good. In case of the isoscalar magnetic moment an exact upper limit is obtained from the maximum eigenvalue of $\frac{1}{2}\left(g^{s}-1\right) S_{z}$ (cf. eq. (3.8)). It appears that the upper limits ohtained from eq. (4.2) are only about $50 \%$ too large.

Assuming that the distribution of the eigenvalues of the operator $O_{00}^{\lambda \tau}$ is also close to gaussian in a space with well-defined angular momentum, one can estimate the magnitude of the fluctuations from the first and second moments of $O_{00}^{\lambda \tau}$. The first moments are given in figs. 3 and 4. The second moments follow from eq. (4.1) and are equal to $\left(\omega_{\max }\right)^{2}$. By the use of condition (4.3) for fixed- $J$ densities $\rho(\omega)$ $J$-dependent upper limits are obtained which are given in the column $\omega_{\max }^{J}$ of tables 
1 and 2 for the $J$-values indicated. In fact we find appreciably smaller values, but in some cases they are still much larger than the shell-model values (see e.g. figs. $2 a$ and $2 b$ ).

Closer upper limits can be obtained by invoking statistical assumptions. If $\left\{\phi_{\alpha}\right\}$ is a complete set of wave functions in the occupation number basis and $\left|\Omega_{i}\right\rangle$ are eigenfunctions of $O_{00}^{\lambda \tau}$ ( $\phi_{\alpha}$ and $\Omega_{i}$ both for the number of active particles under consideration), then one has

$$
\sum_{i}\left\langle\Omega_{i}\left|O_{00}^{\lambda \tau}\right| \Omega_{i}\right\rangle^{2}=\sum_{\alpha}\left\langle\phi_{\alpha}\left|O_{00}^{\lambda \tau}\right| \phi_{\alpha}\right\rangle^{2}+\sum_{\alpha \neq \beta}\left\langle\phi_{\alpha}\left|O_{00}^{\lambda \tau}\right| \phi_{\beta}\right\rangle^{2} .
$$

Because $O_{00}^{\lambda \tau}$ is a one-body operator, there is only a small number $N_{\phi}$ of wave functions $\phi_{\beta}$ which yield a nonzero matrix element for a given $\phi_{\alpha}$. In the case of an E2 (M1) operator one finds $N_{\phi} \approx 6(3)$ for ${ }^{20} \mathrm{Ne}$ and $N_{\phi} \approx 7(4)$ for ${ }^{21} \mathrm{Ne}$. For ${ }^{24} \mathrm{Mg}$ and ${ }^{28} \mathrm{Si}$ one obtains the values $N_{\phi} \approx 9(5)$ and $N_{\phi} \approx 11(7)$, respectively. The nonzero matrix elements are assumed to be distributed according to the gaussian orthogonal ensemble ${ }^{18}$ ). Then the following relations are valid:

$$
\begin{aligned}
& \overline{\left\langle\phi_{\alpha}\left|O_{00}^{\lambda \tau}\right| \phi_{\alpha}\right\rangle^{2}}=2 v^{2} \text {, } \\
& \overline{\left\langle\phi_{\alpha}\left|O_{00}^{\lambda \tau}\right| \phi_{\beta}\right\rangle^{2}}=v^{2} \text { for } \alpha \neq \beta \text {. }
\end{aligned}
$$

The bar denotes ensemble averaging. From eq. (4.5) the ensemble averaged value of the variance of the eigenvalues can be obtained:

$$
\frac{1}{N} \sum_{i}\left\langle\Omega_{i}\left|O_{00}^{\lambda \tau}\right| \Omega_{i}\right\rangle^{2}=2 v^{2}\left(1+\frac{1}{2}\left(N_{\phi}-1\right)\right) .
$$

Hence the eigenvalues of $O_{00}^{\lambda \tau}$ are stretched by a factor $\sqrt{\frac{1}{2}\left(N_{\phi}+1\right)}$ with respect to the diagonal matrix elements, which is the correct asymptotic value for a band matrix having its nonzero and gaussian-distributed elements in $N_{\phi}$ (sub)diagonals ${ }^{18}$ ). The hamiltonian eigenvectors may be assumed to form an arbitrary basis with respect to the operator $O_{00}^{\lambda \tau}$ for which the diagonal elements are not stretched by a factor $>1$ in a transformation to the occupation number basis. Hence the eigenvalues $\left\langle\Omega_{i}\left|O_{00}^{\lambda \tau}\right| \Omega_{i}\right\rangle$ of $O_{00}^{\lambda \tau}$ are stretched by a factor $\sqrt{\frac{1}{2}\left(N_{\phi}+1\right)}$ with respect to the value of the electromagnetic moments. Hence the upper limit is given by

$$
\left|\left\langle\psi_{i}|O| \psi_{i}\right\rangle\right| \leqslant \frac{O_{\max }}{\sqrt{\frac{1}{2}\left(N_{\phi}+1\right)}},
$$

where $O_{\max }$ is the maximum absolute eigenvalue of $O_{00}^{\lambda \tau}$ in the space under consideration.

The upper limits obtained from eq. (4.8) and the upper limits $\omega_{\max }^{J}$ are given in tables 1 and 2 in the column $\omega_{\max }^{r J}$. The upper limits for the quadrupole moments of the states $J=2$ are, even with the inclusion of an effective charge of $\Delta e=0.5 e$ to correct for the finiteness of the sd-shell-model space [see e.g. refs. ${ }^{19.20}$ )], smaller than the experimental values of the lowest-lying states $J=2$. Apparently these 
calculated states do not obey the statistical assumptions; this confirms their collective nature.

Eq. (4.8) with $N_{\phi}$ derived from the occupation number basis, is expected to give good results for the low-lying states which in most cases have large components in this basis. In general the matrix elements $\left\langle\psi_{i}\left|O_{00}^{\lambda \tau}\right| \psi_{j}\right\rangle$ (with $\left\{\psi_{i}\right\}$ cigenfunctions of the hamiltonian) are nonzero in a space with definite angular momentum and isospin for all values of $i$ and $j$, provided, of course, that the angular-momentum selection rules are satisfied.

Making the random-matrix assumptions of eqs. (4.6) then yields a stretching factor of $\sqrt{\frac{1}{2} N}$ with $N$ the dimension of the matrix. Using this factor and the sum rule eq. (4.1) to derive the variance of the fluctuations, one obtains results which compare well with shell-model results for cases in which there is no secular dependence (see figs. $2 \mathrm{a}$ and $2 \mathrm{~b}$ ).

\section{Conclusions}

Shell-model calculations show that electromagnetic moments fluctuate strongly about a linear energy dependence of the secular behaviour. As a consequence it is inevitable to estimate the magnitude of the fluctuations before the use of the secular behaviour (which can be evaluated by means of trace techniques) can be justified for the prediction of electromagnetic moments. The variance of the fluctuations cannot be expressed in terms of traces and therefore their magnitude is estimated by means of upper limits, which can be derived from traces of powers of the electromagnetic operators. In other cases the magnitude of the fluctuations is the most relevant property. The estimation of the upper limits for the fluctuations can be improved if statistical assumptions are made.

This work was performed as part of the research programme of the "Stichting voor Fundamenteel Onderzoek der Materie" (FOM) with financial support from the "Nederlandse Organisatie voor Zuiver-Wetenschappelijk Onderzoek" (ZWO).

\section{Appendix A}

In this appendix a novel method is presented to express fixed- $J T$ traces of an arbitrary tensor operator in terms of fixed $-J_{z} T_{z}$ traces.

Let $O_{\mu}^{\gamma}$ represent a tensor operator with $\gamma$ denoting the quantum numbers of angular momentum $(\lambda)$ and isospin $(\tau)$, and $\mu$ denoting their projections. The trace of $O_{\mu}^{\gamma}$ over states of fixed angular momentum and isospin, $\Gamma$, is determined by the traces over states with fixed projections $\Gamma_{z}$. This can be seen with the help of the following identity:

$$
\operatorname{Tr}_{\Gamma_{z}}\left(O_{0}^{\gamma}\right)=\operatorname{Tr}_{\Gamma=\Gamma_{z}, \Gamma_{z}}\left(O_{0}^{\gamma}\right)+\sum_{\Gamma>\Gamma_{z}} \operatorname{Tr}_{\Gamma \Gamma z}\left(O_{0}^{\gamma}\right)
$$


The ordering of $\Gamma=(J, T)$ is defined as $\Gamma_{1}=\left(J_{1}, T_{1}\right)>\Gamma_{2}=\left(J_{2}, T_{2}\right)$ if $J_{1} \geqslant J_{2}$ and $T_{1} \geqslant T_{2}$ but $\Gamma_{1} \neq \Gamma_{2}$. The traces $\operatorname{Tr}_{\Gamma_{z}}$ in this section are always taken for a fixed number of particles which may be partitioned over different single-particle orbits. For the maximum value of $\Gamma_{z}\left(=\Gamma_{z, \max }\right)$ for the configuration under consideration the second term vanishes and the fixed $-\Gamma \Gamma_{z}$ trace is cqual to the fixcd- $\Gamma_{z}$ tracc. The traces for $\Gamma=\Gamma_{\max }, \Gamma_{z}<\Gamma_{z \text {,max }}$ can now be obtained by application of the WignerEckart theorem. Further application of the Wigner-Eckart theorem and eq. (A.1) yields the traces for $\Gamma<\Gamma_{z}$. Hence, by starting at the maximum value of $\Gamma_{z}$ all fixed $-\Gamma, \Gamma_{z}$ traces can be expressed in terms of fixed- $\Gamma_{z}$ traces by using eq. (A.1) in a recurrent way.

However, the maximum values of the angular momentum and isospin can be much larger than the values for the relevant physical states. Hence, it would be far more economical to solve eq. (A.1) by starting at $\left(\Gamma, \Gamma_{z}\right)=(0,0)$ or $\left(\frac{1}{2}, \frac{1}{2}\right)$.

According to the Wigner-Eckart theorem one has (for reasons of notational simplicity the isospin will not be considered)

$$
\operatorname{Tr}_{J_{z}}\left(O_{0}^{\lambda}\right)=\sum_{J \geqslant J_{z}} \frac{\left\langle J J_{z} \lambda 0 \mid J J_{z}\right\rangle}{\sqrt{2 J+1}} \sum_{\alpha}\left\langle J \alpha\left\|O^{\lambda}\right\| J \alpha\right\rangle
$$

The right-hand side terms for $J=0,1, \ldots,\left[\frac{1}{2}(\lambda-1)\right]$ (with $\left[\frac{1}{2}(\lambda-1)\right]$ denoting the largest integer not larger than $\frac{1}{2}(\lambda-1)$ ) vanish because of angular-momentum selection rules. (Only the case of integer values of the angular momentum $J$ is considered; the case with the half-integer $J$-values can be treated analogously.) The Clebsch-Gordan coefficient in eq. (A.2) is a polynomial of order $\lambda$ in $J_{z}$ with parity $(-1)^{\lambda}$ :

$$
\frac{\left\langle J J_{z} \lambda 0 \mid J J_{z}\right\rangle}{\sqrt{2 J+1}}=\sum_{k=0}^{[12 \lambda]} \alpha_{k}^{\lambda}(J) J_{z}^{\lambda-2 k} .
$$

Hence, after substitution of this polynomial expansion, eq. (A.2) for $J_{z}=$ $(0) 1,, \ldots,\left[\frac{1}{2}(\lambda+1)\right]$ (for odd values of $\lambda$ the equation with $J_{z}=0$ is omitted) constitutes a set of $\left[\frac{1}{2} \lambda\right]+1$ independent linear equations with $\left[\frac{1}{2} \lambda\right]+1$ unknowns:

$$
\beta_{k}^{\lambda} \equiv \sum_{J \geqslant\left[\frac{1}{2}(\lambda+1)\right]} \alpha_{k}^{\lambda}(J) \sum_{\alpha}\left\langle J \alpha\left\|O^{\lambda}\right\| J \alpha\right\rangle, \quad k=0,1, \ldots,\left[\frac{1}{2} \lambda\right] .
$$

Because the value of the determinant for this set of linear equations is equal to

$$
\prod_{1 \leqslant i<j \leqslant[(1(\lambda+1)]}\left(j^{2}-i^{2}\right) \neq 0,
$$

this system can be solved uniquely. The solutions for $\boldsymbol{\beta}_{k}^{\hat{\lambda}}$ are linear combinations of fixed- $J_{z}$ traces. Although in eq. (A.3) the Clebsch-Gordan coefficient is defined for values $J_{z} \leqslant J$ only, the polynomial expansion on the right-hand side does not require this restriction to be meaningful. For values of $J$ obeying the inequalities $J<$ $\left[\frac{1}{2}(\lambda+1)\right]$ and $J_{z} \leqslant J$ the right-hand side of eq. (2.3) is equal to zero. With this observation one can now calculate fixed- $J J_{z}$ traces. 
Substitution of eq. (A.3) into eq. (A.2) yields

$$
\operatorname{Tr}_{J_{z}}\left(O_{0}^{\lambda}\right)=\left\{\sum_{J \geqslant\left[\frac{1}{2}(\lambda+1)\right]}-\sum_{\left[\frac{[}{z}(\lambda+1)\right] \leqslant J<J_{z}}\right\} \sum_{k}^{\left[\frac{[1}{\lambda}\right]} \alpha_{k}^{\lambda}(J) J_{z}^{\lambda-2 k} \sum_{a}\left\langle J \alpha\left\|O^{\lambda}\right\| J \alpha\right\rangle .
$$

This relation can be solved for the (reduced) traces $\sum_{\alpha}\left\langle J \alpha\left\|O^{\lambda}\right\| J \alpha\right\rangle$ successively for increasing values $J_{z}=\left[\frac{1}{2}(\lambda+1)\right]+1,\left[\frac{1}{2}(\lambda+1)\right]+2, \ldots$.

\section{References}

1) J.B. French and K.F. Ratcliff, Phys. Rev. C3 (1971) 94

2) K.F. Ratcliff, Phys. Rev. C3 (1971) 117

3) F.S. Chang, J.B. French and T.H. Thio, Ann. of Phys. 66 (1971) 137

4) C. Jacquemin, Phys. Lett. 43B (1973) 253

5) C. Jacquemin and S. Spitz, Z. Phys. A290 (1979) 251

6) J.J.M. Verbaarschot and P.J. Brussaard, Phys. Lett. 102B (1981) 201

7) J.P. Draayer, J.B. French and S.S.M. Wong, Ann. of Phys. 106 (1977) 472

8) F.S. Chang and J.B. French, Phys. Lett. 44B (1973) 131

9) P.J. Brussaard and P.W.M. Glaudemans, Shell-model applications in nuclear spectroscopy (NorthHolland, Amsterdam, 1977)

10) A. de-Shalit and I. Talmi, Nuclear shell theory (Academic Press, New York, 1963) p. 234

11) J.N. Ginocchio and S. Ayik, Nucl. Phys. A239 (1975) 365

12) J.B. French, in Many-body description of nuclei and reactions, Int. School of Physics, Enrico Fermi, course 36, ed. C. Bloch (Academic Press, New York, 1966) p. 278

13) J.N. Ginocchio, Phys. Rev. C8 (1973) 135

14) J.J.M. Verbaarschot, Ph.D. thesis, University of Utrecht (1982)

15) J.P. Draayer, J.B. French and S.S.M. Wong, Ann. of Phys. 106 (1977) 503

16) T.R. Halemane, Phys. Lett. 108B (1982) 256

17) T.R. Halemane and J.B. French, Phys. Rev. C25 (1982) 2029

18) C.E. Porter, Statistical theory of spectra: fluctuations (Academic Press, New York, 1965)

19) L.S. Hsu, Phys. Lett. 25B (1967) 558

20) B.C. Metsch and P.W.M. Glaudemans, Z. Phys. 307 (1982) 251 